The online version of this article is published within an Open Access environment subject to the conditions of the Creative Commons Attribution-NonCommercial-ShareAlike licence $<$ http://creativecommons.org/licenses/by-nc-sa/3.0/>. The written permission of Cambridge University Press must be obtained for commercial re-use.

\title{
Analytic moduli for unfoldings of germs of generic analytic diffeomorphisms with a codimension $k$ parabolic point
}

\author{
C. ROUSSEAU \\ DMS and CRM, Université de Montréal, C.P. 6128, succ. Centre-ville, Montréal, \\ Quebec, H3C 3J7, Canada \\ (e-mail: rousseac@dms.umontreal.ca)
}

(Received 13 March 2012 and accepted in revised form 17 April 2013)

\begin{abstract}
In this paper we provide a complete modulus of analytic classification for germs of generic analytic families of diffeomorphisms which unfold a parabolic fixed point of codimension $k$. We start by showing that a generic family can be 'prepared', i.e. brought to a prenormal form $f_{\epsilon}(z)$ in which the multi-parameter $\epsilon$ is almost canonical (up to an action of $\mathbb{Z} / k \mathbb{Z}$ ). As in the codimension one case treated in P. Mardešić, R. Roussarie and C. Rousseau [Modulus of analytic classification for unfoldings of generic parabolic diffeomorphisms. Mosc. Math. J. 4 (2004), 455-498], we show that the Ecalle-Voronin modulus can be unfolded to give a complete modulus for such germs. For this purpose, we define unfolded sectors in $z$-space that constitute natural domains on which the map $f_{\epsilon}$ can be brought to normal form in an almost unique way. The comparison of these normalizing changes of coordinates on the different sectors forms the analytic part of the modulus. This construction is performed on sectors in the multi-parameter space $\epsilon$ such that the closure of their union provides a neighborhood of the origin in parameter space.
\end{abstract}

\section{Introduction}

This paper is part of a large program to classify and understand the finite codimension singularities of analytic dynamical systems and their unfoldings. The first tool for this purpose is to look at normal forms. A certain number of singularities have normal forms depending on a finite number of parameters which are formal invariants of the system. In a series of papers $[1,6,7,11,13,15,16]$ we have developed the program for singularities with common features: their normal forms have a finite number of formal parameters, called formal invariants, and there exist formal changes of coordinates to normal form which are divergent and $k$-summable in some sense. The singularities studied come 
from the merging of $k+1$ simple singularities $([\mathbf{1}, \mathbf{6}, \mathbf{7}, \mathbf{1 1}]$ when $k=1$, and $[\mathbf{1 6}]$ for a codimension $k$ saddle-node of a planar vector field), or from the merging of a singularity with a periodic orbit ([15] and [13]). Examples of such singularities are the fixed points of a one-dimensional diffeomorphism with resonant multiplier (the multiplier is a root of unity), the saddle-nodes, the resonant saddles (the quotient of eigenvalues is a negative rational number) or the weak foci of a two-dimensional vector field, and the irregular nonresonant singularities of a linear differential system.

The meaning of the formal invariants is best understood when one unfolds the system. Indeed, in all cases except [6], there are natural canonical parameters which are analytic invariants for the family. The formal invariants unfold analytically in the parameters. In all generic cases (simple singularities), the sum of the number of parameters and of the number of formal invariants is exactly equal to the sum of the number of (linear) invariants at each simple singular point in the unfolding (multipliers or eigenvalues).

The analytic classification of germs of generic analytic families unfolding these singularities is done by giving a complete modulus of analytic classification for each family. The modulus comprises a formal part described above, and an analytic part which is given by the unfolding of the analytic part of the modulus of the singularity itself. To describe the modulus, we cover a fixed neighborhood of the singularity with $2 k$ sectors depending on the parameters. On each sector, we construct normalizing changes of coordinates to the normal form. The sectors are such that the normalizing changes of coordinates are almost unique (up to symmetries of the normal form on the full neighborhood). The comparison of the normalizing changes of coordinates on the intersections of the sectors provides the modulus. The construction can be performed depending analytically on the parameters on some sectors in parameter space, the closure of which covers a full neighborhood of the origin in parameter space. The analytic part of the modulus of the singularity is the limit of the analytic part of the modulus of the unfolding: this allows us to interpret the modulus of the codimension $k$ singularity and to understand why it is so complex. Indeed, there are rigid models at each unfolded singularity which are glued in a non-trivial way. The modulus at the limit is the limit of these non-trivial gluings. Also, the unfolded singularities can be resonant for sequences of parameter values converging to the origin, in which case they themselves have formal invariants. The limits of these formal invariants are also encoded in the analytic part of the modulus of the limit singularity: this is the parametric resurgence phenomenon discussed in $\S 6$.

In this paper, we build a complete modulus of analytic classification for germs of generic analytic diffeomorphisms with a codimension $k$ parabolic point. As a first step, in $\S 2$, we show that the family can be prepared, i.e. brought through a change of variable and parameter to a form in which the parameters are analytic invariants up to an action of $\mathbb{Z} / k \mathbb{Z}$. There is a brief description of notation in $\S 3$. In $\S 4$, we then describe the sectors in the variable and in the parameter space, and we construct the Fatou coordinates which normalize the diffeomorphism on the different sectors. The comparison of the Fatou coordinates yields the analytic part of the modulus. This analytic part depends analytically on the parameters on each sector in parameter space. In $\$ 5$, we then show that the modulus we have defined is a complete modulus of analytic classification, namely 
that two families with the same modulus are analytically conjugate, with the conjugacy depending analytically on the parameters. In $\S 6$, we briefly discuss the interpretation of the modulus in the parametric resurgence phenomenon.

The next step in this program is to identify precisely the set of realizable moduli. For this purpose, it is necessary to identify the sufficient conditions for a modulus to be realized as the modulus of a germ of analytic family unfolding a diffeomorphism with a parabolic point of codimension $k$. We hope to address this question in a forthcoming paper.

\section{Preparation of the family}

Definition 2.1. A germ of an analytic $k$-parameter family $f_{\eta}$ unfolding a diffeomorphism of the form

$$
f_{0}(z)=z+z^{k+1}+o\left(z^{k+1}\right)
$$

can be written (modulo a translation) in the form

$$
f_{\eta}(z)=z+p_{\eta}(z)(1+O(|(z, \eta)|))
$$

where

$$
p_{\eta}(z)=z^{k+1}+c_{k-1}(\eta) z^{k-1}+\cdots+c_{1}(\eta) z+c_{0}(\eta) .
$$

It is called a germ of generic analytic k-parameter family if the mapping $\eta \mapsto$ $\left(c_{0}(\eta), \ldots c_{k-1}(\eta)\right)$ is a germ of analytic diffeomorphism from $\left(\mathbb{C}^{k}, 0\right)$ to $\left(\mathbb{C}^{k}, 0\right)$.

THEOREM 2.2. For any germ of generic analytic k-parameter family $f_{\eta}$ unfolding a diffeomorphism of the form (2.1) there exists a germ of change of coordinate and parameter to a prepared form

$$
\bar{f}_{\epsilon}(Z)=Z+P_{\epsilon}(Z)\left(1+Q_{\epsilon}(Z)+P_{\epsilon}(Z) R_{\epsilon}(Z)\right)
$$

with

$$
P_{\epsilon}(z)=Z^{k+1}+\epsilon_{k-1} Z^{k-1}+\cdots+\epsilon_{1} Z+\epsilon_{0}
$$

such that:

- $Q_{\epsilon}(Z)=c_{k}(\epsilon) Z^{k}+\cdots+c_{1}(\epsilon) Z+c_{0}(\epsilon)$, with $c_{0}(0)=\cdots=c_{k-1}(0)=0$;

- if $Z_{1}, \ldots, Z_{k+1}$ are the fixed points of $\bar{f}_{\epsilon}$, if $\lambda_{j}(\epsilon)$ is defined by $\lambda_{j}(\epsilon)=\bar{f}_{\epsilon}^{\prime}\left(Z_{j}\right)$, if the germ of analytic function a $(\epsilon)$ is defined through

$$
\sum 1 / \ln \left(\lambda_{j}(\epsilon)\right)=a(\epsilon)
$$

when the $Z_{j}$ are distinct, and the vector field $v_{\epsilon}$ is defined by

$$
v_{\epsilon}(Z)=\frac{P_{\epsilon}(Z)}{1+a(\epsilon) Z^{k}} \frac{\partial}{\partial Z},
$$

then

$$
\ln \lambda_{j}(\epsilon)=\ln \left(\bar{f}_{\epsilon}^{\prime}\left(Z_{j}\right)\right)=v_{\epsilon}^{\prime}\left(Z_{j}\right) .
$$

Moreover, the parameters $\epsilon$ are almost unique in the following sense. If $\tilde{f}_{\tilde{\epsilon}}(\widetilde{Z})$ is another prepared form for the same unfolding $f_{\eta}$, then there exists $\tau$ such that $\tau^{k}=1$ and

$$
\left(\widetilde{Z}, \tilde{\epsilon}_{k-1}, \ldots, \tilde{\epsilon}_{0}\right)=\left(\tau Z+o(Z), \tau^{2} \epsilon_{k-1}, \tau^{3} \epsilon_{k-2}, \ldots, \epsilon_{1}, \tau \epsilon_{0}\right) .
$$


Proof. Using a translation in $z$ and a change of parameters, we can suppose that the fixed points of $f_{\eta}$ are given by the zeros of

$$
p_{\eta}(z)=z^{k+1}+\eta_{k-1} z^{k-1}+\cdots+\eta_{1} z+\eta_{0} .
$$

Hence, we can write the unfolding in the form

$$
f_{\eta}(z)=z+p_{\eta}(z)\left(1+m_{\eta}(z)\right)
$$

with $m_{\eta}(z)=O(\eta, z)$. Using the Weierstrass division theorem on $m_{\eta}$ allows us to write $f_{\eta}$ in the form

$$
f_{\eta}(z)=z+p_{\eta}(z)\left(1+q_{\eta}(z)+p_{\eta}(z) n_{\eta}(z)\right)
$$

with $q_{\eta}(z)$ a polynomial of degree $k$.

Let $z_{1}, \ldots, z_{k+1}$ be the fixed points of $f_{\eta}$. There exists a polynomial $S_{\eta}(z)$ of degree $\leq k$ such that at the points $z_{j}$ we have

$$
\ln f_{\eta}^{\prime}\left(z_{j}\right)=p_{\eta}^{\prime}\left(z_{j}\right)\left(1+S_{\eta}\left(z_{j}\right)\right) .
$$

Such a polynomial is found by the Lagrange interpolation formula using

$$
S_{\eta}\left(z_{j}\right)=\frac{\ln \left(1+p_{\eta}^{\prime}\left(z_{j}\right)\left(1+q_{\eta}\left(z_{j}\right)\right)\right)}{p_{\eta}^{\prime}\left(z_{j}\right)}-1 .
$$

Indeed, the formula works for distinct $z_{j}$. The limit exists when two fixed points coalesce (codimension one case). We can fill in for the other values of $\eta$ by Hartogs's theorem.

By Kostov's theorem [5], there exists a local analytic change of coordinate and parameters transforming the vector field

$$
v_{1, \eta}(z)=p_{\eta}(z)\left(1+S_{\eta}(z)\right) \frac{\partial}{\partial z}
$$

into

$$
v_{\epsilon}(Z)=P_{\epsilon}(Z) /\left(1+a(\epsilon) Z^{k}\right) \frac{\partial}{\partial Z},
$$

where $P_{\epsilon}$ is defined in (2.3). We apply this change of coordinate and parameter to $f_{\eta}$.

Claim. This brings $f_{\eta}$ to a prepared form $\bar{f}_{\epsilon}$. Indeed, it sends the zeros $z_{j}$ of $p_{\eta}(z)$ to the zeros $Z_{j}$ of $P_{\epsilon}(Z)$. Since the $z_{j}$ are the fixed points of $f_{\eta}$, their images are the fixed points $Z_{j}$ of $\bar{f}_{\epsilon}$. So

$$
\bar{f}_{\epsilon}(Z)=Z+P_{\epsilon}(Z) G_{\epsilon}(Z)=Z+P_{\epsilon}(Z)\left(1+Q_{\epsilon}(Z)+P_{\epsilon}(Z) L_{\epsilon}(Z)\right) .
$$

Let $Z_{j}$ be a fixed point of $\bar{f}_{\epsilon}$. Then, from (2.8),

$$
f_{\eta}^{\prime}\left(z_{j}\right)=\exp \left(p^{\prime}\left(z_{j}\right)\left(1+S_{\eta}\left(z_{j}\right)\right)=\exp \left(v_{1, \eta}^{\prime}\left(z_{j}\right)\right)\right.
$$

Since multipliers at fixed points of diffeomorphisms (respectively eigenvalues at singular points of vector fields) are invariant under change of coordinates, we then have

$$
\bar{f}_{\epsilon}^{\prime}\left(Z_{j}\right)=\lambda_{j}(\epsilon)=f_{\eta}^{\prime}\left(z_{j}\right)=\exp \left(v_{1, \eta}^{\prime}\left(z_{j}\right)\right)=\exp \left(v_{\epsilon}^{\prime}\left(Z_{j}\right)\right)
$$


which is what we need for a prepared family. Moreover, we see that

$$
\bar{f}_{\epsilon}^{\prime}\left(Z_{j}\right)=1+P_{\epsilon}^{\prime}\left(Z_{j}\right)\left(1+Q_{\epsilon}\left(Z_{j}\right)\right)
$$

can be calculated from $P_{\epsilon}$ and $Q_{\epsilon}$ alone.

We now need to show that $Q_{0}(Z)=O\left(Z^{k}\right)$, so we consider the particular case where $\epsilon_{j}=0$ for $j \neq 1$. The fixed points are then $Z_{0}=0$, and $Z_{1}, \ldots, Z_{k}$, with $Z_{j}^{k}+\epsilon_{1}=0$. This yields $P_{\epsilon}^{\prime}(0)=\epsilon_{1}$ and $P_{\epsilon}\left(Z_{j}\right)=-k \epsilon_{1}$ for $j>1$. On one side, we need to have

$$
\bar{f}_{\epsilon}^{\prime}\left(Z_{j}\right)=\exp \left(\frac{-k \epsilon_{1}}{1-a(\epsilon) \epsilon_{1}}\right)
$$

which yields $\bar{f}_{\epsilon}^{\prime}\left(Z_{j}\right)=1-k \epsilon_{1}+O\left(\epsilon_{1}^{2}\right)$. On the other side, we also have $\bar{f}_{\epsilon}^{\prime}\left(Z_{j}\right)=$ $1-k \epsilon_{1}\left(1+Q_{\epsilon}\left(Z_{j}\right)\right)$. Hence, if the coefficient $c_{\ell}(\epsilon)$ of $Z^{\ell}$ in $Q_{\epsilon}$ was such that $c_{\ell}(0) \neq 0$ for $\ell<k$, and $\ell$ was minimum with this property, then this would yield $\bar{f}_{\epsilon}^{\prime}\left(Z_{j}\right)=$ $1-k \epsilon_{1}+C \epsilon_{1}^{1+\ell / k}$ with $C \neq 0$, hence a contradiction.

We now show that the parameters are almost canonical. For that purpose, let us consider a prepared family, which we denote $f_{\epsilon}(z)$ (forgetting the bar). We will prove that the only freedom we have in the choice of a prepared family is a change

$$
\left(z, \epsilon_{k-1}, \epsilon_{k-2} \ldots, \epsilon_{0}\right) \mapsto\left(\tau z+o(z), \tau^{-2} \epsilon_{k-1}, \tau^{-3} \epsilon_{k-2}, \ldots, \tau \epsilon_{0}\right),
$$

where $\tau=\exp (2 \pi i \ell / k), \ell=0, \ldots, k-1$. Indeed, let us suppose that two prepared families $f_{\epsilon}(z)$ and $\tilde{f}_{\tilde{\epsilon}}(\tilde{z})$ are conjugate under a map $(\tilde{\epsilon}, \tilde{z})=\left(g(\epsilon), h_{\epsilon}(z)\right): \tilde{f}_{g(\epsilon)}=h_{\epsilon} \circ$ $f_{\epsilon} \circ h_{\epsilon}^{-1}$. The fixed points $z_{j}$ (respectively $\tilde{z}_{j}$ ) of the family $f_{\epsilon}$ (respectively $\tilde{f}_{\tilde{\epsilon}}$ ) are the same as the singular points of a vector field $P_{\epsilon}(z) /\left(1+a(\epsilon) z^{k}\right) \partial / \partial z=v_{\epsilon}(z)$ (respectively $\left.\tilde{P}_{\tilde{\epsilon}}(\tilde{z}) /\left(1+\tilde{a}(\tilde{\epsilon}) \tilde{z}^{k}\right) \partial / \partial \tilde{z}=\tilde{v}_{\tilde{\epsilon}}(\tilde{z})\right)$. Note that $a(\epsilon)=\tilde{a}(g(\epsilon))$. Then $h_{\epsilon}$ sends the fixed points $z_{j}$ to the fixed points $\tilde{z}_{j}$. Hence, $h_{\epsilon}^{*}\left(\tilde{v}_{g(\epsilon)}\right)(z)=P_{\epsilon}(z) U_{\epsilon}(z) \partial / \partial z=\bar{v}_{\epsilon}(z)$, where $U_{\epsilon} \neq 0$. Moreover, the vector fields $v_{\epsilon}$ and $\bar{v}_{\epsilon}$ have the same singular points with the same eigenvalues. Hence, we can write

$$
\bar{v}_{\epsilon}=P_{\epsilon}(z)\left(\frac{1}{1+a(\epsilon) z^{k}}+P_{\epsilon}(z) M_{\epsilon}(z)\right) \frac{\partial}{\partial z}=v_{\epsilon}\left(1+P_{\epsilon}(z) N_{\epsilon}(z)\right) \frac{\partial}{\partial z} .
$$

There exists a change of coordinate $K_{\epsilon}$ such that $K_{\epsilon}^{*}\left(v_{\epsilon}\right)=\bar{v}_{\epsilon}$. Such a change of coordinate is given by the flow of $v_{\epsilon}$ under some time $T_{\epsilon}$ (see, for instance, [16, Corollary 8.2]): $K_{\epsilon}=\Phi_{v_{\epsilon}}^{T_{\epsilon}}$, where $T_{\epsilon}$ is a solution of

$$
v_{\epsilon}\left(T_{\epsilon}\right)=-\frac{P_{\epsilon}(z) N_{\epsilon}(z)}{1+P_{\epsilon}(z) N_{\epsilon}(z)}
$$

which obviously has an analytic solution. Then $K_{\epsilon}^{-1} \circ h_{\epsilon}^{*}\left(\tilde{v}_{g(\epsilon)}\right)=v_{\epsilon}$. The result follows from the following theorem proved in [16].

THEOREM 2.3. [16] We consider two vector fields, $v_{\epsilon}=P_{\epsilon}(z) /\left(1+a(\epsilon) z^{k}\right) \partial / \partial z$ and $\tilde{v}_{\tilde{\epsilon}}=\tilde{P}_{\tilde{\epsilon}}(\tilde{z}) /\left(1+\tilde{a}(\tilde{\epsilon}) \tilde{z}^{k}\right) \partial / \partial \tilde{z}$, which are conjugate in a neighborhood of the origin containing all the singular points. Then, necessarily the conjugacy between $v_{\epsilon}$ and $\tilde{v}_{\tilde{\epsilon}}$ is of the form (2.9) with $\tau$ some kth root of unity. 


\section{Remark 2.4.}

(1) The meaning of the formal invariant $a$ now becomes clear. The polynomial $P_{\epsilon}$ has $k+1$ roots and depends on $k$ parameters only. An additional parameter is needed so that the eigenvalues at the singular points of $v_{\epsilon}$ are independent.

(2) In a prepared family the parameters are almost canonical. This simplifies greatly the problem of classifying germs of families under analytic equivalence. Indeed, in general, two prepared families $f_{\epsilon}(z)$ and $\tilde{f}_{\tilde{\epsilon}}(\tilde{z})$ are analytically conjugate under an analytic diffeomorphism $(\tilde{\epsilon}, \tilde{z})=\left(g(\epsilon), h_{\epsilon}(z)\right)$ such that $\tilde{f}_{g(\epsilon)}=h_{\epsilon} \circ f_{\epsilon} \circ h_{\epsilon}^{-1}$. Here, we can limit ourselves to maps $g(\epsilon)=\left(\tau^{-2} \epsilon_{k-1}, \tau^{-3} \epsilon_{k-2}, \ldots, \tau \epsilon_{0}\right)$ for $\tau$ some $k$ th root of unity. Note also that the parameters of the family of diffeomorphisms $f_{\epsilon}$ are the same as those of the vector field $v_{\epsilon}$.

\section{Some preliminaries and notation}

The vector field $w_{\epsilon}=P_{\epsilon}(z) \partial / \partial z$ is invariant under

$$
\left(z, t, \epsilon_{k-1}, \ldots, \epsilon_{0}\right) \mapsto\left(c z, c^{-k} t, c^{2} \epsilon_{k-1}, \ldots, c^{k+1} \epsilon_{0}\right) .
$$

This suggests the following norm in $\epsilon$-space

$$
\|\epsilon\|:=\max \left(\left|\epsilon_{k-1}\right|^{1 / 2}, \ldots,\left|\epsilon_{0}\right|^{1 /(k+1)}\right),
$$

and the following equivalence relation in $\epsilon$-space

$$
\epsilon \simeq \epsilon^{\prime} \Longleftrightarrow \text { there exists } c \in \mathbb{R}^{+},\left(\epsilon_{k-1}^{\prime}, \ldots, \epsilon_{0}^{\prime}\right)=\left(c^{2} \epsilon_{k-1}, \ldots, c^{k+1} \epsilon_{0}\right) .
$$

We define

$$
\mathbb{D}_{\rho}=\{\epsilon ;\|\epsilon\| \leq \rho\} .
$$

Remark 3.1. We should normally describe the modulus on an open polydisk. We prefer to work with the closed polydisk $\mathbb{D}_{\rho}$, which allows us to use its conic structure under the equivalence relation (3.2). Whenever we consider $\mathbb{D}_{\rho}$ it is implicitly meant that the family is defined for $\epsilon$ in a larger open polydisk.

We consider the open component $\Sigma_{0}$ of $\mathbb{D}_{\rho}$ which is the set of $\epsilon$ for which the discriminant $\Delta(\epsilon)$ of $P_{\epsilon}$ is non-zero, i.e. the vector field is non-degenerate. It is a union of curves $\left\{\left(c^{2} \epsilon_{k-1}, \ldots, c^{k+1} \epsilon_{0}\right) \mid c \in(0,1)\right\}$ for some $\epsilon$ with $\|\epsilon\|=\rho$, which are invariant under the equivalence relation $\simeq$.

Notation 3.2. We denote by $T_{C}$ the translation by $C$

$$
T_{C}(Z)=Z+C
$$

\section{The construction of Fatou coordinates}

From now on we will limit ourselves to a prepared family

$$
f_{\epsilon}(z)=z+P_{\epsilon}(z)\left(1+Q_{\epsilon}(z)+P_{\epsilon}(z) R_{\epsilon}(z)\right) .
$$

Let us first discuss the general strategy. In general, there are obstructions to conjugating a germ of analytic family of the type (4.1) with its normal form. So the idea is to construct 
the conjugacies with the normal form on sectorial domains in $z$-space. These sectorial domains (there will be $2 k$ of them) should be sufficiently large for the conjugacies to be almost unique. Then, because of the (almost) uniqueness, their comparison measures the obstruction to an analytic conjugacy with the normal form on a full neighborhood $\mathbb{D}_{r}$ of the origin in $z$-space, and it is an analytic invariant for the system. But we also want the conjugacies to depend analytically on $\epsilon$. So we want our sectors in $z$-space and the conjugacies defined on them to depend analytically on $\epsilon$. There are obstructions to doing this, because the sectors are attached both to some fixed part of the boundary of $\mathbb{D}_{r}$ and to some fixed points which may rotate around each other when the parameters vary. Hence the construction can only be done analytically in $\epsilon$ for $\epsilon$ in some sectors in parameter space.

The analytic classification of analytic diffeomorphisms (or their unfoldings) is closely related to the embedding problem, which consists in determining when an analytic diffeomorphism is embeddable in a flow, i.e. conjugate to the time one map of a vector field. Since the normal form of $f_{\epsilon}$ is the time one map of the one-dimensional vector field $v_{\epsilon}$, this is the case when the diffeomorphism or family of diffeomorphisms is conjugate to its normal form. A standard practice in the field is to change coordinate to a coordinate in which the normal form is just the translation by 1 , which we call $T_{1}$ (this coordinate is nothing other than the time of the vector field). Hence a Fatou coordinate is just a diffeomorphism conjugating the system to $T_{1}$. But, in practice, we will also change coordinate for $f_{\epsilon}$.

4.1. Construction of the sectors in z-space. We want to describe the dynamics of the family $f_{\epsilon}$ over a fixed neighborhood $\mathbb{D}_{r}$ of the origin for all values of the parameters in a polydisk $\mathbb{D}_{\rho}$. The construction of the $2 k$ sectors for the different values of the parameters in a sectorial domain in parameter space is very similar to the construction of the squid sectors in [16]; in particular, quantitative versions of the qualitative constructions described below appear there. We recall the construction for the purpose of completeness. It was only done for values of $\epsilon$ inside $\Sigma_{0}$ (i.e. the fixed points are distinct (regular)), with continuous limit at $\epsilon=0$, but it can easily be extended to all values of the parameters. For $\epsilon \in \Sigma_{0}$ (respectively $\epsilon \notin \Sigma_{0}$ ), each sector in $z$-space is adherent to two regular fixed points (respectively two not necessarily distinct regular or singular fixed points), i.e. the closure of the sector contains the fixed points.

The formal normal form of $f_{\epsilon}$ is the time one map of the vector field $v_{\epsilon}$. For small $z$ and $\epsilon$, this vector field is close to the simpler vector field

$$
w_{\epsilon}=P_{\epsilon}(z) \frac{\partial}{\partial z} .
$$

The phase portrait of $w_{\epsilon}$ has been extensively studied in the literature (see, for instance, [2]), and we will be brief with the details. It is organized by its pole at infinity (see Figure 1(a)), which has order $k-1$ (hence for $k=1$, infinity is a regular point). The pole has $2 k$ separatrices which generically land on a singular point. In $z$-space we choose $r$ sufficiently small for the dynamics of $f_{0}$ to be as in Figure 1(b); this comes from the fact that germs of diffeomorphisms of the form $f_{0}(z)=z+z^{k+1}+o\left(z^{k+1}\right)$ are topologically equivalent to the time one map of the flow of the vector field $z^{k+1} \partial / \partial z$ (see, for instance, 


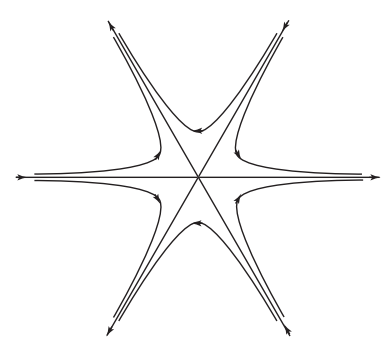

(a) Neighborhood of infinity

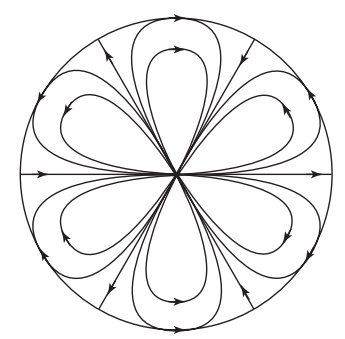

(b) $w_{0}$ on $\mathbb{D}_{r}$

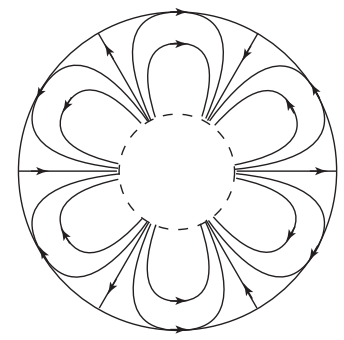

(c) Near $\partial d \mathbb{D}_{r}$

FIGURE 1 . The phase portrait of $w_{\epsilon}$ at infinity and near the boundary of $\mathbb{D}_{r}$ when $k=3$.

[4, Theorem 2]). We then choose $\rho$ sufficiently small for all fixed points of $f_{\epsilon}$ to remain inside $\mathbb{D}_{r}$, and the dynamics of $f_{\epsilon}$ to be as in Figure 1(c) along the boundary. For that purpose, we will in particular require that

$$
\rho<\frac{r^{k}}{2},
$$

a condition that will be assumed throughout the paper. We will allow further restriction of $\rho$.

To construct normalizing changes of coordinates, it is standard to change coordinate system and to work in the time coordinate of the vector field $w_{\epsilon}$. This change of coordinate system is given by

$$
Z=p_{\epsilon}^{-1}(z)=\int_{\infty}^{z} \frac{d z}{P_{\epsilon}(z)} .
$$

In particular,

$$
Z=p_{\epsilon}^{-1}(z)= \begin{cases}\sum_{j=1}^{k+1} \frac{1}{P_{\epsilon}^{\prime}\left(z_{j}\right)} \ln \left(z-z_{j}\right), & z_{j} \text { distinct } \\ -\frac{1}{k z^{k}}, & \epsilon=0 .\end{cases}
$$

In the $Z$-coordinate, the map $f_{\epsilon}$ is transformed into a map $F_{\epsilon}$ which is close to $T_{1}$, the translation by 1 .

PROPOSITION 4.1. The function $F_{\epsilon}$ is a small perturbation of the translation $T_{1}$ in the $C^{1}$-topology. More precisely, there exists $K>0$ such that, for $r>0$ and $\rho>0$ sufficiently small with $\rho$ satisfying condition (4.3),

$$
\begin{gathered}
\left|F_{\epsilon}(Z)-Z-1\right|<K r, \\
\left|F_{\epsilon}^{\prime}(Z)-1\right|<K r^{k+1} .
\end{gathered}
$$

Proof. This kind of estimate is standard (see for instance [17]). For the sake of completeness we include the proof. On $\partial \mathbb{D}_{r}$ for small $\epsilon, p_{\epsilon}^{-1}$ is a small perturbation of $-1 / k z^{k}$. Hence (4.5) is satisfied for $|z|=r$ and $\epsilon$ sufficiently small. Since the function $\left(F_{\epsilon}(Z)-Z-1\right) \circ p_{\epsilon}^{-1}$ is uniform in $\mathbb{D}_{r}$ and bounded at the fixed points, by the maximum principle (4.5) is satisfied everywhere for the same values of $\epsilon$. 
Moreover, if we let $z=p_{\epsilon}(Z)$, then

$$
F_{\epsilon}^{\prime}(Z)=\left(p_{\epsilon}^{-1}\right)^{\prime}\left(f_{\epsilon}(z)\right) \cdot f_{\epsilon}^{\prime}(z) \cdot p_{\epsilon}^{\prime}(Z)=\frac{P_{\epsilon}(z)}{P_{\epsilon}\left(f_{\epsilon}(z)\right)} f_{\epsilon}^{\prime}(z) .
$$

Note that this function is analytic in $(z, \epsilon)$ since $P_{\epsilon}$ vanishes at the fixed points of $f_{\epsilon}$. Now let us consider the case $\epsilon=0$. Since

$$
\begin{gathered}
F_{0}^{\prime}(Z)-1=\frac{P_{0}(z) f_{0}^{\prime}(z)-P_{0}\left(f_{0}(z)\right)}{P_{0}\left(f_{0}(z)\right)}, \\
P_{0}\left(f_{0}(z)\right)=z^{k+1}+(k+1) z^{2 k+1}+O\left(z^{2 k+2}\right)
\end{gathered}
$$

and

$$
P_{0}(z) f_{0}^{\prime}(z)=z^{k+1}+(k+1) z^{2 k+1}+O\left(z^{2 k+2}\right),
$$

then $F_{0}^{\prime}(Z)-1=O\left(z^{k+1}\right)$, yielding $\left|F_{0}^{\prime}(Z)-1\right|<(K / 2) r^{k+1}$ for $r$ sufficiently small. The estimate (4.6) will then be valid for $\|\epsilon\|<\rho$ with $\rho$ sufficiently small.

The map $p_{\epsilon}$ is a $k$-sheeted covering of $\mathbb{C} \backslash\left\{z_{1}, \ldots, z_{k+1}\right\}$. The inverse image of $\mathbb{C} \mathbb{P}^{1} \backslash \mathbb{D}_{r}$ is given by sequences of equidistant holes in the different sheets. Making a turn around one hole corresponds to making $(1 / k)$ th of a turn around $\partial \mathbb{D}_{r}$. Each outgoing separatrix of infinity undergoes a homoclinic connection with an incoming separatrix of infinity when the vector field $w_{\epsilon}$ is transformed into a vector field $w_{\epsilon}(\theta)=e^{i \theta} w_{\epsilon}$ for some $\theta$ to be discussed later. The distance between the centers of two consecutive holes corresponds to $e^{-i \theta} T$, where $T \in \mathbb{R}^{+}$is the travel time along the homoclinic loop of $w_{\epsilon}(\theta)$ (a pole is reached in finite time). This distance is also equal to $\pm \sum_{j \in I}\left(2 \pi i / P_{\epsilon}^{\prime}\left(x_{j}\right)\right)$, where $I \subset\{1, \ldots, k+1\}$ is the set of indices of the singular points on one side of the homoclinic loop of $w_{\epsilon}(\theta)$. We will describe these distances in $\S 4.2$ after we have described the sectors in $\epsilon$-space covering $\Sigma_{0}$.

To simplify the presentation, it will suffice to construct the modulus for parameter values inside $\Sigma_{0}$ (i.e. the fixed points are distinct). Note that $\Sigma_{0}=\{\epsilon \mid \Delta(\epsilon) \neq 0\}$, where $\Delta(\epsilon)$ is the discriminant of $P_{\epsilon}$. Since the construction will be bounded near the points of $\Delta=0$, it will be possible to fill the holes later. The sectors will be bounded by four curves:

(i) a portion $S$ of $\partial \mathbb{D}_{r}$ of opening $\beta \in(\pi / k, 3 \pi / 2 k)$,

(ii) two 'complex time trajectories' of $w_{\epsilon}$ starting at the end points of $S$ and ending in two distinct singular points,

(iii) a 'complex time trajectory' of $w_{\epsilon}$ joining the two singular points inside $\mathbb{D}_{r}$.

The curves can be chosen so that any three sectors have a void intersection.

The idea is that the sectors should contain representatives of a large set of orbits of $f_{\epsilon}$ parameterized by $\mathbb{C}^{*}$. Since the linear maps are the only holomorphic diffeomorphisms of $\mathbb{C}^{*}$, this endows the set of orbits of $f_{\epsilon}$ over the sector of a conformal structure with a very rigid coordinate system. To ensure that this is true, it is best if the boundaries of the sectors are transversal to curves invariant under $f_{\epsilon}$. The simplest idea is to take trajectories of the orthogonal vector field $i w_{\epsilon}$ starting on $\partial \mathbb{D}_{r}$ and corresponding to vertical lines in the Z-coordinate for (ii) and (iii). This was done by Oudkerk in [9]. However, for some values of $\epsilon$ for which $i w_{\epsilon}$ is not far from having a homoclinic loop, there may exist no such trajectories inside $\mathbb{D}_{r}$. In that case, we replace each trajectory of $i w_{\epsilon}$ by a union of two 

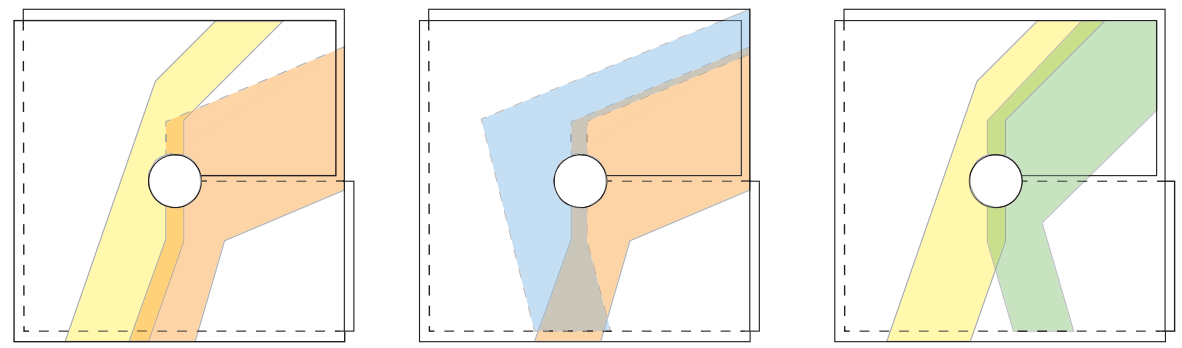

FIGURE 2. Different pairs of sets in Z-space corresponding to four sectors in $z$-space for the same value of $\epsilon$.

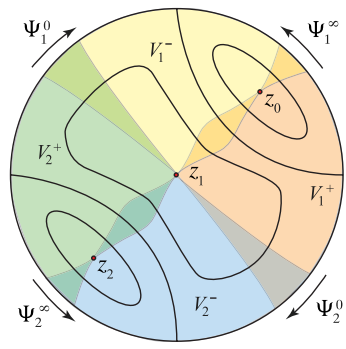

(a) A first set of sectors

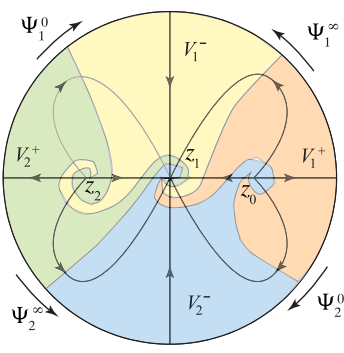

(b) A second set of sectors

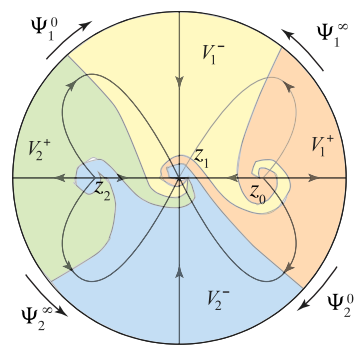

(c) Another set of sectors for the same $\epsilon$ as in (b)

FIGURE 3. Different types of sets of sectors in $z$-space. In (b) and (c) their overlapping has not been drawn.

to three trajectories of $e^{i \theta} w_{\epsilon}$ for some $\theta \in[\pi / 4,3 \pi / 4]$ (it could be a different $\theta$ on each different piece of the trajectories). Figure 2 (respectively Figure 3 ) represents such sectors in $Z$-space (respectively $z$-space).

When considering limits for $\epsilon \rightarrow 0$, it will suffice to consider such limits along the curves in parameter space that are invariant under the equivalence relation $\simeq$ defined in (3.2). In the limit for $\epsilon=0$, the sectors will be given by half-planes in $Z$-space limited by vertical lines.

In practice, when we consider $\epsilon$ in an invariant curve in parameter space, we want any compact set in the limit sector at $\epsilon=0$ to be included in the corresponding sector for $\epsilon$ sufficiently small. For this purpose, each complex time trajectory of (ii) will be given by a finite piece of $i w_{\epsilon}$ starting on $\partial \mathbb{D}_{r}$ followed by a trajectory of $e^{i \theta} w_{\epsilon}$ ending in the singular point, for some $\theta \in[\pi / 4,3 \pi / 4]$ (it could be a different $\theta$ at each singular point). The trajectory in (iii) is a union of trajectories of $i w_{\epsilon}$ and of the two $e^{i \theta} w_{\epsilon}$ used in the neighborhood of the singular points. It is the same $\theta$ that is used for all curves approaching a given singular point. Such sectors are best visualized in $Z$-space where they are bounded by vertical segments and segments, lines or half-lines making some angle $\theta \in[\pi / 4,3 \pi / 4]$ with the horizontal direction (Figure 2).

Remark 4.2. The construction can also be carried out for values of $\epsilon \notin \Sigma_{0}$, for which $\Delta(\epsilon)=0$. In that case, for some sectors the singular points of (ii) are identical and there is no trajectory of type (iii). In practice these sectors correspond to domains conformally 


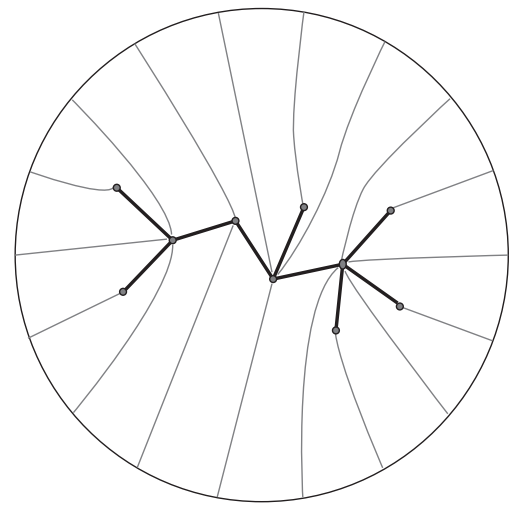

(a)

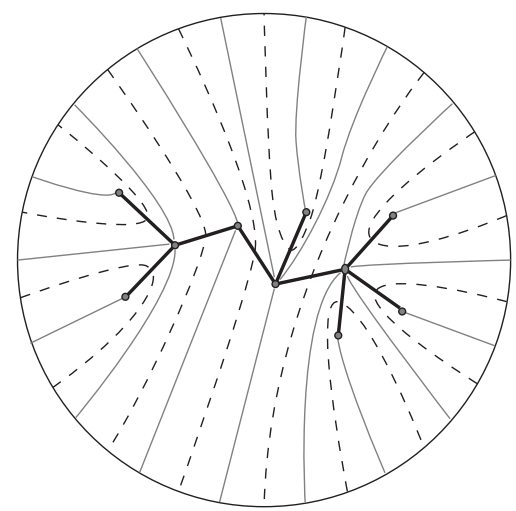

(b)

FIGURE 4. The graph (tree) formed by the separatrices of infinity ending at the singular points, together with trajectories joining the singular points. The distances between holes on the different sheets in $Z$-space are given by the complex travel times from $\infty$ to $\infty$ along the dotted lines in (b).

equivalent to a half-plane in $Z$-space in a leaf where there is no sequence of holes (the distance between holes has become infinite).

Notation 4.3. The $2 k$ sectors in $z$-space are called $V_{j}^{ \pm}$(or $V_{j, \epsilon}^{ \pm}$). The sectors $V_{j}^{+}$ (respectively $V_{j}^{-}$) are centered on regions of $\partial \mathbb{D}_{r}$ where $f_{\epsilon}$ exits (respectively enters) $\mathbb{D}_{r}$.

It remains to discuss how to choose the angles $\theta$. For this, we need to make a digression in parameter space.

4.2. The sectors in parameter space. We first consider the case where $r=\infty$. Then, the construction described above can be done with $\theta=\pi / 2$, except when $i w_{\epsilon}$ has a homoclinic trajectory: this occurs when there exists a non-trivial subset $I \subset\{1, \ldots, k+1\}$ such that $\sum_{j \in I}\left(i / P_{\epsilon}^{\prime}\left(x_{j}\right)\right) \in i \mathbb{R}$. The closure of this set in parameter space contains $\Delta=0$. This can also be seen geometrically, since there is always an attracting and a repelling separatrix of infinity ending at a multiple point. The complement of the closure of this set in parameter space is a union of $C(k)=(1 /(k+1))\left(\begin{array}{c}2 k \\ k\end{array}\right)$ connected components $\widetilde{W}_{\ell}$. This comes from the following theorem which should be applied to $i w_{\epsilon}$ after a scaling in $z$.

THEOREM 4.4. In the parameter space of polynomial vector fields of the form $P_{\epsilon}(z) \partial / \partial z$ there exist exactly $C(k)=(1 /(k+1))\left(\begin{array}{c}2 k \\ k\end{array}\right)$ different connected components of generic vector fields with simple singular points and no homoclinic loops. These components are simply connected.

Proof. This is easily derived from [2]. There, it is shown that there are exactly $C(k)$ combinatorial invariants describing how the separatrices of generic vector fields are attached to the singular points (see Figure 4). It suffices to show that each such combinatorial invariant corresponds to a simply connected open set in $\epsilon$-space. Indeed, each vector field is characterized by its combinatorial invariant plus $k$ analytic invariants $b_{j} \in \mathbb{C}$, such that $\operatorname{Im} b_{j}>0$, corresponding to the transit time from $\infty$ to $\infty$ along the 
dotted lines in Figure 4(b). These times are periods that can be calculated using the residue theorem. Each such vector field is realized on an abstract manifold $M_{\epsilon}$ constructed by adequately sewing $k+1$ horizontal strips, the width of which are given by the invariants $b_{j}$. Hence, the family $M_{\epsilon}$, for $\epsilon$ in a connected component, is parameterized by $\mathcal{B}=$ $\left\{\mathbf{b}=\left(b_{1}, \ldots, b_{k}\right) \in \mathbb{H}^{k}\right\}$. This set is obviously simply connected. On a given connected component, we have a holomorphic bijection $\epsilon \mapsto \mathbf{b}$, yielding that the construction of $M_{\epsilon}$ depends holomorphically on $\mathbf{b} \in \mathcal{B}$. Hence, any connected component of generic vector fields in parameter space has the same topology as $\mathbb{H}^{k}$.

Sectors providing a covering of $\Sigma_{0}$. The sectors $\left\{W_{\ell} \mid \ell=1, \ldots, C(k)\right\}$ in parameter space provide a covering of $\Sigma_{0}$. Each sector $W_{\ell}$ has a vertex at $\epsilon=0$, and is an enlargement of the corresponding $\widetilde{W}_{\ell}$. Let us call $\Sigma_{1}$ the subset of $\Sigma_{0}$ for which $i w_{\epsilon}$ is generic, i.e. has no homoclinic trajectory. In practice, we will not be able to use the trajectories of $i w_{\epsilon}$ for all values of $\epsilon \in \widetilde{W}_{\ell}$. Indeed, it may happen that a separatrix of infinity makes large spirals disconnecting $\mathbb{D}_{r}$ before landing at a singular point. If that is the case, then we replace part of the corresponding trajectory of $i w_{\epsilon}$ by a trajectory of $e^{i \theta(\epsilon)} w_{\epsilon}$ for some $\theta(\epsilon)$ with $\theta(\epsilon) \in(\pi / 4,3 \pi / 4)$ depending continuously on $\epsilon$. Not only can this be done for $\epsilon \in \widetilde{W}_{\ell}$, but we can also extend the sectors $\widetilde{W}_{\ell}$ so that their union covers $\Sigma_{0}$. Indeed, the boundaries of $\widetilde{W}_{\ell}$ consist of hypersurfaces where homoclinic connections occur. For each homoclinic connection, we choose an appropriate angle $\theta$ to destroy it. In particular, the sign of $\theta-\pi / 2$ is such that the respective positions of the separatrices are the same as for $\widetilde{W}_{\ell}$ (this is ensured by the continuity of $\theta(\epsilon)$ ). Each $\widetilde{W}_{\ell}$ contains a codimension $k$ subset for which $k$ homoclinic loops of $w_{\epsilon}$ (not $i w_{\epsilon}$ !) appear simultaneously. On this subset, which is the organizing center of $W_{\ell}$, we use trajectories of $i w_{\epsilon}$.

Remark 4.5. The distances of the holes are well defined for $\epsilon$ in some $\widetilde{W}_{\ell}$; these distances are vectors. They are precisely $-i$ times the complex travel time along the trajectories in the dotted lines of Figure 4(b) for the vector field $i w_{\epsilon}$. There are $k$ such times, one for each sheet in $Z$-space. For $\epsilon \in W_{\ell}$, we extend these distances continuously by considering $e^{-i \theta(\epsilon)}$ times the complex travel time along corresponding trajectories in dotted lines for the vector field $e^{i \theta(\epsilon)} w_{\epsilon}$, where $\theta(\epsilon)$ depends continuously on $\epsilon$.

Note that for $\epsilon \in W_{\ell} \cap W_{\ell^{\prime}}$ and $k>2$ we may have distinct sets of distances, depending on whether we consider the $W_{\ell}$ or $W_{\ell^{\prime}}$ point of view.

\subsection{Construction of the Fatou coordinates. The construction is performed for $\epsilon \in W_{\ell}$.}

Definition 4.6. We define a translation domain as the saturation by $F_{\epsilon}$ of any preimage by $p_{\epsilon}$ of a sector in $z$ as described in $\$ 4.1$, provided that the $Z$-domain is sufficiently wide to contain a strip, $\hat{C}(\ell)$, bounded by a curve $\ell$ going from $\operatorname{Im}(Z)=-\infty$ to $\operatorname{Im}(Z)=+\infty$, with slope greater than 1 at all points, and its image by $F_{\epsilon}$ (see Figure 5). The curve $\ell$ is called an admissible curve for the translation domain, which we will denote by $\mathcal{T}_{\epsilon}$.

The Fatou coordinates are changes of coordinates on the translation domains conjugating the diffeomorphism $F_{\epsilon}$ to the translation by 1 , denoted $T_{1}$.

THEOREM 4.7. There exist $r>0$ and $\rho>0$ sufficiently small with $\rho<r^{k} / 2$ so that for $\epsilon \in \mathbb{D}_{\rho}$, for any translation domain $\mathcal{T}_{\epsilon}$, and $Z_{0}(\epsilon) \in \mathcal{T}_{\epsilon}$, the following hold. 


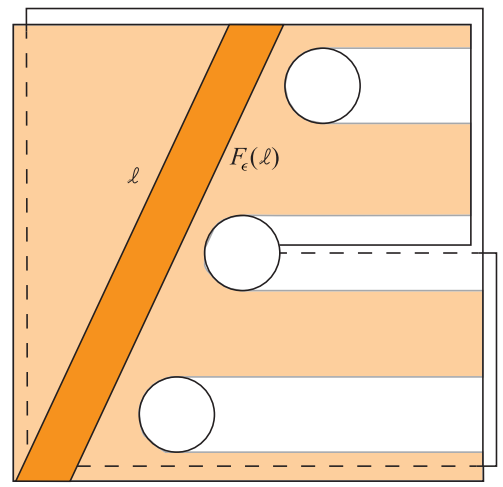

FIGURE 5. A translation domain.

(1) There exists a holomorphic diffeomorphism $\Phi_{\epsilon}: \mathcal{T}_{\epsilon} \rightarrow \mathbb{C}$, such that

$$
\Phi_{\epsilon} \circ F_{\epsilon}=T_{1} \circ \Phi_{\epsilon},
$$

for $Z \in \mathcal{T}_{\epsilon} \cap F_{\epsilon}^{-1}\left(\mathcal{T}_{\epsilon}\right)$. Moreover, $\lim _{\operatorname{Im}(Z) \rightarrow \pm \infty} \operatorname{Im}\left(\Phi_{\epsilon}(Z)\right)= \pm \infty$.

(2) If $\Phi_{1, \epsilon}$ and $\Phi_{2, \epsilon}$ are two solutions of (4.7), then there exists $A \in \mathbb{C}$ such that $\Phi_{2, \epsilon}(Z)=T_{A} \circ \Phi_{1, \epsilon}(Z)$. In particular, if $Z_{0}(\epsilon) \in \mathcal{T}_{\epsilon}$, there exists a unique holomorphic diffeomorphism $\Phi_{\epsilon}$ satisfying (4.7) together with $\Phi_{\epsilon}\left(Z_{0}(\epsilon)\right)=0$.

(3) If $Z_{0}(\epsilon)$ depends analytically on $\epsilon$ with limit $Z_{0}(0)$ at $\epsilon=0$, then the unique Fatou coordinate $\Phi_{\epsilon}$ satisfying $\Phi_{\epsilon}\left(Z_{0}(\epsilon)\right)=0$ depends analytically on $\epsilon \in W_{\ell}$. For $\epsilon \rightarrow 0$, it converges to $\Phi_{0}$, uniformly on compact sets in $\mathcal{T}_{0}$.

Proof. The proof is exactly the same as in [7] since it relies only on Proposition 4.1. We put it here for the sake of completeness. The technique we use is identical to that of Shishikura [17], as adapted in [7]. It consists in constructing a quasi-conformal conjugacy of $F_{\epsilon}$ to $T_{1}$, and then using Ahlfors-Bers' theorem to transform it into a conformal conjugacy.

Throughout the proof we do not mention the $\epsilon$-dependence. Let $\ell$ be an admissible curve for the translation domain $\mathcal{T}$, and $\hat{C}(\ell)$ the corresponding strip. Let $\beta(t), t \in \mathbb{R}$, be a parametrization of $\ell$ such that $\operatorname{Im}(\beta(t))=t$. We define $h_{1}: C_{0}=\left\{(X, Y) \in \mathbb{R}^{2} \mid 0 \leq\right.$ $X \leq 1\} \rightarrow \hat{C}(\ell)$ by:

$$
h_{1}(X, Y)=(1-X)(\beta(Y))+X F_{\epsilon}(\beta(Y)) .
$$

Then

$$
\begin{aligned}
& \frac{\partial h_{1}}{\partial X}=F_{\epsilon}(\beta(Y))-\beta(Y), \\
& \frac{\partial h_{1}}{\partial Y}=\beta^{\prime}(Y)\left[1+X\left(F_{\epsilon}^{\prime}(\beta(Y))-1\right)\right] .
\end{aligned}
$$

Using the estimates (4.5) and (4.6), these formulas imply that

$$
\frac{\partial h_{1}}{\partial X}-1=u(X, Y), \quad \frac{\partial h_{1}}{\partial Y}-\beta^{\prime}(Y)=v(X, Y) \quad \text { with }|u|<K r,|v| \leq 2 K r^{k+1} \text {. }
$$


Let $\mu_{h_{1}}=\left(\partial h_{1} / \partial \bar{Z}\right) /\left(\partial h_{1} / \partial Z\right)$ be the dilatation coefficient field of $h_{1}$. One has

$$
\frac{\partial h_{1}}{\partial \bar{Z}}=\frac{1}{2}\left[1+u+i\left(\beta^{\prime}(Y)+v\right)\right] \quad \text { and } \quad \frac{\partial h_{1}}{\partial Z}=\frac{1}{2}\left[1+u-i\left(\beta^{\prime}(Y)+v\right)\right] .
$$

Note that $\beta(Y)=\gamma(Y)+i Y$, where $\gamma^{\prime}(Y) \in[-1,1]$. We have

$$
\mu_{h_{1}}=\frac{u+i v+i \gamma^{\prime}(Y)}{\left(2-i \gamma^{\prime}(Y)\right)+u-i v}<\frac{1+K r+K r^{k+1}}{1-K r-K r^{k+1}}<1
$$

when $r$ is sufficiently small, independently of $\epsilon$ such that $\|\epsilon\| \leq \rho<r^{k} / 2$.

Hence, $h_{1}$ is a quasi-conformal mapping on the strip $C_{0}$ and satisfies $h_{1}^{-1}\left(F_{\epsilon}(Z)\right)=$ $h_{1}^{-1}(Z)+1$ for $Z \in \ell$. Moreover, $\mu=\mu_{h_{1}}$ is a Beltrami field on $C_{0}$. (One can also write that $\mu=h_{1}^{*} \mu_{0}$, where $\mu_{0}$ is the standard Beltrami field on $\mathbb{C}$ (defined by the function 0 ).)

We extend $\mu$ to a Beltrami field on all of $\mathbb{C}$ by means of the translation $T_{1}$. Indeed, the extended $\mu$ is periodic of period 1 , is in $L^{\infty}(\mathbb{C})$ and has an $L^{\infty}$-norm: $\|\mu\|_{\infty}=$ $\left\|\mu_{h_{1}}\right\|_{\infty}<1$.

The universal covering

$$
w=E(W)=\exp (-2 \pi i W)
$$

from $\mathbb{C}$ to $\mathbb{C}^{*}$ induces a holomorphic diffeomorphism from $\mathbb{C} / T_{1}$ to $\mathbb{C}^{*}$. Since $\mu$ is invariant by $T_{1}$, the map $E$ induces a Beltrami field $\tilde{\mu}$ on $\mathbb{C}^{*}$ with the same norm: $\mu=E^{*}(\tilde{\mu})$. We can extend $\tilde{\mu}$ to a Beltrami field on the Riemann sphere $\mathbb{C P}^{1}$ by letting, for instance, $\tilde{\mu}(0)=\tilde{\mu}(\infty)=0$.

By Ahlfors-Bers' measurable mapping theorem there exists a unique quasi-conformal mapping $\tilde{h}_{2}: \mathbb{C P}^{1} \rightarrow \mathbb{C P}^{1}$ such that $\tilde{h}_{2}^{*} \mu_{0}=\tilde{\mu}$, and $\tilde{h}_{2}(0)=0, \tilde{h}_{2}(\infty)=\infty, \tilde{h}_{2}(1)=1$. Since $0,1 \in E^{-1}(1)$, this map lifts to a quasi-conformal map $h_{2}: \mathbb{C} \rightarrow \mathbb{C}$ sending 0 to 0 , and 1 to 1 . Indeed, one can lift $\tilde{h}_{2}$ to a map $h_{2}$ such that $h_{2}(0)=0$. The circle in $\mathbb{C P}^{1}$ which turns one time around 0 or $\infty$ lifts into the line segment $[0,1]$ in $\mathbb{C}$. This means that $h_{2}(1)=1$. We also have that $\operatorname{Im}\left(h_{2}(X+i Y)\right) \rightarrow \pm \infty$ when $Y \rightarrow \pm \infty$.

Moreover, $h_{2}$ commutes with $T_{1}$. Indeed, the homeomorphism $H_{2}=h_{2} \circ T_{1} \circ h_{2}^{-1}$ induces the identity on $\mathbb{C P}^{1}$ and must then be a power of the deck transformation $T_{1}$ of the universal covering map $E$, i.e. $H_{2}=T_{1}^{n}$ for some $n \in \mathbb{Z}$. Now, $H_{2}(0)=h_{2} \circ T_{1}(0)=$ $h_{2}(1)=1$. This forces $n=1$, and then $H_{2}=T_{1}$, i.e. $h_{2} \circ T_{1}=T_{1} \circ h_{2}$.

We define $\phi: \hat{C}(\ell) \rightarrow \mathbb{C}$ by $\phi=h_{2} \circ h_{1}^{-1}$, and extend it by $T_{1}$ to a mapping $\phi: \mathbb{C} \rightarrow \mathbb{C}$ which is quasi-conformal and preserves the standard conformal structure. Hence, it is conformal. For $Z \in \ell$, one has $T_{1} \circ \phi(Z)=\phi \circ F_{\epsilon}(Z)$. Then $\phi$ extends to a map $\Phi$ of $\mathcal{T}$ into $\mathbb{C}$ by $\Phi(Z)=\phi \circ F_{\epsilon}^{n}(Z)-n$, where $n \in \mathbb{Z}$ is such that $F_{\epsilon}^{n}(Z) \in \hat{C}(\ell)$. This map $\Phi$ is a holomorphic diffeomorphism which verifies $\Phi \circ F_{\epsilon}=T_{1} \circ \Phi$.

If $\Phi_{i, \epsilon}, i=1,2$, satisfy (4.7), let $\chi_{1, \epsilon}=\Phi_{2, \epsilon} \circ\left(\Phi_{1, \epsilon}\right)^{-1}$ and $\chi_{2, \epsilon}=\Phi_{1, \epsilon} \circ\left(\Phi_{2, \epsilon}\right)^{-1}$. Both $\chi_{j, \epsilon}$ satisfy $\chi_{j, \epsilon}(W+1)=\chi_{j, \epsilon}(W)+1$ and this relation allows us to extend them to global diffeomorphisms of $\mathbb{C}$ commuting with $T_{1}$. It follows that $\Phi_{2, \epsilon} \circ \Phi_{1, \epsilon}^{-1}$ is a translation.

By construction, it is standard that the Fatou coordinate $\Phi_{\epsilon}$ depends analytically on $\epsilon$, with uniform convergence to $\Phi_{0}$ on compact sets on $\mathcal{T}_{0}$ when $\epsilon \rightarrow 0$. 


\subsection{Components of the analytic part of the modulus and normalization.}

Notation 4.8. We denote by $\mathcal{T}_{j, \epsilon}^{ \pm}$the translation domain containing $p_{\epsilon}^{-1}\left(V_{j, \epsilon}^{ \pm}\right)$. We define $\Phi_{j, \epsilon}^{ \pm}$as the Fatou coordinate on $\mathcal{T}_{j, \epsilon}^{ \pm}$containing $p_{\epsilon}^{-1}\left(V_{j, \epsilon}^{ \pm}\right)$.

We have $2 k$ strips given by $p_{\epsilon}^{-1}\left(V_{j, \epsilon}^{ \pm}\right)$inside the translation domains. These strips intersect. For $\epsilon \in \Sigma_{0}$, each strip intersects one or two of its neighbors along two semiinfinite strips. Altogether, this yields $2 k$ semi-infinite strips. Let us call $\Phi_{j, \epsilon}^{+}$(respectively $\Phi_{j, \epsilon}^{-}$) the Fatou coordinates corresponding to translation domains on the left (respectively right) of the principal hole as in Figure 2 (the Fatou coordinate $\Phi_{j, \epsilon}^{ \pm}$is defined on a translation domain $\mathcal{T}_{j, \epsilon}^{ \pm}$containing $p_{\epsilon}^{-1}\left(V_{j, \epsilon}^{ \pm}\right)$).

Comparison of the Fatou coordinates. For this purpose we introduce the maps

$$
\left\{\begin{array}{l}
\Psi_{j, \epsilon}^{0}=\Phi_{j, \epsilon}^{-} \circ\left(\Phi_{j+1, \epsilon}^{+}\right)^{-1}, \\
\Psi_{j, \epsilon}^{\infty}=\Phi_{j, \epsilon}^{-} \circ\left(\Phi_{j, \epsilon}^{+}\right)^{-1} .
\end{array}\right.
$$

Note that there exists $Y_{0}>0$ such that $\Psi_{j, \epsilon}^{0}$ (respectively $\Psi_{j, \epsilon}^{\infty}$ ) is defined in a region $\operatorname{Im}(Z)<Y_{0}$ (respectively $\operatorname{Im}(Z)>Y_{0}$ ). These maps are almost the analytic part of the modulus, which will indeed almost be given by a collection of $2 k$ families of analytic maps. But, the maps $\Psi_{j, \epsilon}^{0}$ and $\Psi_{j, \epsilon}^{\infty}$ are only defined up to composition with left and right translations. So the modulus will be given by the corresponding quotient. It is useful to work with representatives that depend nicely on the parameters, and in particular have a limit when $\epsilon \rightarrow 0$ inside a sector $W_{\ell}$. For that purpose, we introduce a normalization.

LEMMA 4.9. The transition maps $\Psi_{j, \epsilon}^{0, \infty}, j=1, \ldots, k$, can be expanded as Fourier series with constant terms $A_{j, \epsilon}^{0, \infty}$. It is possible to choose the base points of the Fatou coordinates in Theorem 4.7 so that, for all $j, A_{j, \epsilon}^{0}=-A_{j, \epsilon}^{\infty}=\pi i a(\epsilon) / k$.

Proof. The proof of this fact is the same as in [15].

This leaves one degree of freedom in the choice of one base point which can be chosen depending analytically on $\epsilon$. We use it in the following way: we choose one base point $z_{0} \epsilon$ $V_{1}^{+} \subset \mathbb{D}_{r}$ which is a regular point; for instance, $z_{0}=3 r / 4$. Let $Z_{0}(\epsilon)=p_{\epsilon}^{-1}\left(z_{0}\right) \in \mathcal{T}_{1, \epsilon}^{+}$. We normalize the first Fatou coordinate so that $\Phi_{1, \epsilon}^{+}\left(Z_{0}(\epsilon)\right)=0$. Then the only freedom of choice is the base point $z_{0} \in V_{1}^{+}:$it is now independent of $\epsilon$.

Definition 4.10. A set of Fatou coordinates is called a normalized set of Fatou coordinates if for all $j$, the constant terms $A_{j, \epsilon}^{0, \infty}$ of $\Psi_{j, \epsilon}^{0, \infty}$ satisfy $A_{j, \epsilon}^{0}=-A_{j, \epsilon}^{\infty}=\pi i a(\epsilon) / k$. If, furthermore, there exists $z_{0} \in \mathbb{D}_{r}$ which, for all $\epsilon \in \mathbb{D}_{\rho}$, is a regular point of $f_{\epsilon}$ inside $V_{1, \epsilon}^{+}$, and such that $\Phi_{1, \epsilon}^{+}\left(p_{\epsilon}^{-1}\left(z_{0}\right)\right)=0$, then the set of Fatou coordinates is said to be strongly normalized.

\section{The theorem of analytic classification}

Notation 5.1. For each sectorial domain $W_{\ell}$ in $\Sigma_{0}$, we denote by $\Psi_{j, \epsilon, \ell}^{0, \infty}$ the elements of the corresponding analytic part of the modulus. 
THEOREM 5.2. Two germs of generic prepared families $f_{\epsilon}$ and $\tilde{f}_{\tilde{\epsilon}}$ unfolding a germ of diffeomorphism with a parabolic point of codimension $k$ are analytically conjugate if and only if they have the same normalized modulus, i.e. there exists $m \in \mathbb{Z}_{k}$ such that:

- $\quad$ for all $j=0, \ldots, k-1, \tilde{\epsilon}_{j}=\exp (-2 \pi i m(j-1) / k) \epsilon_{j}$, and $a(\epsilon)=\tilde{a}(\tilde{\epsilon})$;

- $\quad$ for each sectorial domain $W_{\ell}$ in $\Sigma_{0}$, there exists $C_{\ell}(\epsilon)$ depending analytically on $\epsilon \in W_{\ell}$, with $C_{\ell}(0)$ independent of $\ell$, such that for $j=1, \ldots, k$

$$
\Psi_{j, \epsilon, \ell}^{0, \infty}=T_{-C_{\ell}(\epsilon)} \circ \widetilde{\Psi}_{j+m, \epsilon, \ell}^{0, \infty} \circ T_{C_{\ell}(\epsilon)} .
$$

In particular, a germ of generic prepared family $f_{\epsilon}$ unfolding a germ of diffeomorphism with a parabolic point of codimension $k$ is analytically equivalent to its normal form if and only if, for all $j=1, \ldots, k$ and all $\epsilon \in \Sigma_{0}, \Psi_{j, \epsilon}^{0}=T_{\pi i a(\epsilon) / k}, \Psi_{j, \epsilon}^{\infty}=T_{-\pi i a(\epsilon) / k}$.

Proof. Suppose that the two prepared families $f_{\epsilon}$ and $\tilde{f}_{\tilde{\epsilon}}$ are analytically conjugate. The first condition (formal part) follows from Theorem 2.2. The canonical parameters are either the same, or such that $\tilde{\epsilon}_{j}=\exp (-2 \pi i m(j-1) / k) \epsilon_{j}$ for some fixed $m$, and we can of course take $\rho$ sufficiently small that the moduli of $f_{\epsilon}$ and $\tilde{f}_{\tilde{\epsilon}}$ are defined for $\epsilon \in \mathbb{D}_{\rho}$. Let $h_{\epsilon}$ be a conjugacy between $f_{\epsilon}$ and $\tilde{f}_{\tilde{\epsilon}}$, such that $h_{\epsilon} \circ f_{\epsilon}=\tilde{f}_{\tilde{\epsilon}} \circ h_{\epsilon}$. Then $H_{\epsilon}=p_{\tilde{\epsilon}} \circ h_{\epsilon} \circ p_{\epsilon}^{-1}$ is a conjugacy between $F_{\epsilon}$ and $\widetilde{F}_{\tilde{\epsilon}}$, yielding that $\Phi_{j, \epsilon}^{0, \infty} \circ H_{\epsilon}^{-1}$ is a Fatou coordinate for $\tilde{f}_{\tilde{\epsilon}}$ such that $\Phi_{j, \epsilon}^{0, \infty} \circ H_{\epsilon}^{-1}\left(p_{\epsilon}^{-1}\left(z_{0}\right)\right)=0$. The result follows by (almost) uniqueness properties of Fatou coordinates.

Conversely, consider two families with the same normalized moduli. We can always conjugate the second family with a rotation and suppose that the two families have the same canonical parameter $\epsilon$. Then, for $\epsilon \in W_{\ell}$, a conjugacy between the two families is given by

$$
h_{\epsilon, \ell}=p_{\epsilon} \circ\left[\left(\widetilde{\Phi}_{j, \epsilon, \ell}^{ \pm}\right)^{-1} \circ T_{C_{\ell}(\epsilon)} \circ \Phi_{j, \epsilon, \ell}^{ \pm}\right] \circ p_{\epsilon}^{-1}, \quad z \in V_{j, \epsilon, \ell}^{ \pm} .
$$

It is well defined because the two families have the same modulus, and it depends analytically on $\epsilon \in W_{\ell}$. We first show that in the generic case where the modulus is nontrivial (i.e. at least one component $\Psi_{j, \epsilon}^{0, \infty}$ is not affine) the $h_{\epsilon, \ell}$ are independent of $\ell$, and hence glue in a uniform $h_{\epsilon}$ on $\Sigma_{0}$. Since, by construction, $h_{\epsilon}$ is bounded near $\{\Delta(\epsilon)=0\}$, it can be extended to $\mathbb{D}_{\rho}$. We then show how to treat the non-generic case.

So, let us try to show that $h_{\epsilon, \ell}$ and $h_{\epsilon, \ell^{\prime}}$ coincide for $\epsilon \in W_{\ell} \cap W_{\ell^{\prime}}$ and see what condition is necessary for this. We have that $g_{\epsilon}=\left(h_{\epsilon, \ell^{\prime}}\right)^{-1} \circ h_{\epsilon, \ell}$ is a symmetry of the first family; it commutes with $f_{\epsilon}$. The families of diffeomorphisms commuting with $f_{\epsilon}$ are determined exactly as in the case $\epsilon=0$, and the result is the same; they are given by powers $f_{\epsilon}^{\alpha(\epsilon)}$ of $f_{\epsilon}$, where:

- $\alpha(\epsilon) \in \mathbb{C}$, if all $\Psi_{j, \epsilon}^{0, \infty}$ are translations;

- $\alpha(\epsilon) \in \mathbb{Z}$, when the only translations $T_{b}$ which commute with all $\Psi_{j, \epsilon}^{0, \infty}$ are the translations with $b \in \mathbb{Z}$;

- $\quad \alpha(\epsilon) \in(1 / n) \mathbb{Z}$, when the only translations $T_{b}$ which commute with all $\Psi_{j, \epsilon}^{0, \infty}$ are the translations with $b \in(1 / n) \mathbb{Z}$ for some $n \in \mathbb{N}^{*}$.

(Full details for the argument in the case $k=1$ can be found in [10].) In the two last cases the symmetries are discrete, yielding that $\alpha(\epsilon)$ is constant. Since $C_{\ell}(0)$ is independent of $\ell$ and all $\Psi_{j, \epsilon, \ell}^{0, \infty}$ have the same limit $\Psi_{j, 0}^{0, \infty}$ independent of $\ell$, then $g_{0}=\mathrm{id}$, yielding that $\alpha(\epsilon) \equiv 0$. 
In the non-generic case of a trivial modulus, i.e. when all $\Psi_{j, \epsilon, \ell}^{0, \infty}$ are translations, then the modulus is invariant under conjugacy with a translation. So we can of course suppose that the moduli of $f_{\epsilon}$ and $\tilde{f}_{\tilde{\epsilon}}$ are strongly normalized. Also, because the components of the modulus are translations we can obviously take $C_{\ell}(\epsilon) \equiv 0$, which implies that $h_{\epsilon, \ell}\left(z_{0}\right)=h_{\epsilon, \ell^{\prime}}\left(z_{0}\right)=\tilde{z}_{0}$ for all $\ell$, i.e. $g_{\epsilon}\left(z_{0}\right)=z_{0}$. Then, necessarily $\alpha(\epsilon) \equiv 0$.

\section{Geometric interpretation and parametric resurgence phenomenon}

The codimension one case is very different from the codimension $k$ case, where $k>1$. Indeed, in the codimension one case, the analytic part of the modulus contains two functions, $\Psi_{\epsilon}^{0}$ and $\Psi_{\epsilon}^{\infty}$, and there are two fixed points. The generic case is the case where the two fixed points have multipliers with norm different from 1 and hence are linearizable, yielding that the system can be brought to the normal form in the neighborhood of each fixed point. In that case, the non-triviality of the modulus (i.e. at least one of $\Psi_{\epsilon}^{0}$ or $\Psi_{\epsilon}^{\infty}$ is not a translation) expresses the fact that the two normalizing changes of coordinates do not match. Note that the domains of $\Psi_{\epsilon}^{0}$ or $\Psi_{\epsilon}^{\infty}$ correspond to sectors in $z$-space attached to exactly one fixed point (see, for instance, [3] and [8]). So $\Psi_{\epsilon}^{0}$ is attached to one fixed point, and $\Psi_{\epsilon}^{\infty}$ to the other. When the norm of the multiplier at one fixed point is equal to 1 , then the linearizability or nonlinearizability of the fixed point can be determined from the $\Psi_{\epsilon}^{0}$ or $\Psi_{\epsilon}^{\infty}$ which is attached to it. The parametric resurgence phenomenon is the phenomenon where the nonlinearizability of a fixed point for sequences of resonant values of $\epsilon$ (i.e. values of $\epsilon$ such that the multiplier at one fixed point is a root of unity) converging to the origin can be decided directly from $\Psi_{0}^{0}$ or $\Psi_{0}^{\infty}$. We will explain it in more generality below.

When $k>1$, then $k+1 \neq 2 k$, and we lose the fact that exactly one component $\Psi_{j, \epsilon}^{0}$ or $\Psi_{j, \epsilon}^{\infty}$ of the modulus is attached to each fixed point. So the $2 k$ components of the modulus should rather be considered as attached to the $2 k$ sectors on the boundary, when we normalize the diffeomorphism from the boundary of $\partial \mathbb{D}_{r}$. What remains is that the $\Psi_{j, \epsilon}^{0}$ and $\Psi_{j, \epsilon}^{\infty}$ are attached to the fixed points in a surjective way. Moreover, all components attached to a fixed point are of the same type, either all $\Psi_{j, \epsilon}^{0}$, or all $\Psi_{j, \epsilon}^{\infty}$.

In $\$ 4.4$ we discussed the fact that the strips given by $p_{\epsilon}^{-1}\left(V_{j, \epsilon}^{ \pm}\right)$intersect along half strip(s). But in Figure 3 we also see some intersection parts that go from one fixed point to another. These are given by the projections of infinite strips in $Z$-space. These infinite strips are only obtained when one considers the periodicity in $Z$-space. The $k$ different periods are given by the $k$ different distances between holes. Then, an infinite strip is obtained by the intersection of one strip with the translation by a period of a second strip. On an infinite strip, the comparison of two Fatou coordinates is just a translation $T_{L(\epsilon)}(W)=W+L(\epsilon)$, called a Lavaurs translation, with $L(\epsilon) \rightarrow \infty$ when $\epsilon \rightarrow 0$. This comes from the fact that the comparison is a diffeomorphism commuting with $T_{1}$ on an infinite strip from $\operatorname{Im}(W)=-\infty$ to $\operatorname{Im}(W)=\infty$.

Now, to describe the dynamics near a fixed point, we use the renormalized return map; this map is defined on the orbit space, or its covering space, namely the $Z$-variable. It is the composition of the transition maps encountered when making one turn in the positive direction around the fixed point. These transition maps are the $\Psi_{j, \epsilon}^{0}$ or the $\Psi_{j, \epsilon}^{\infty}$ attached to the fixed point, alternating with as many Lavaurs translations. 
It is easier to visualize the renormalized return maps when changing to the coordinate

$$
w=\exp (-2 \pi i Z) \text {. }
$$

The maps $\Psi_{j, \epsilon}^{0, \infty}$ expressed in this coordinate will be denoted $\psi_{j, \epsilon}^{0, \infty}$. The Lavaurs translations become Lavaurs linear maps $L_{c(\epsilon)}(w)=c(\epsilon) w$. Then the renormalized return map has a fixed point at 0 (respectively $\infty$ ) when built with $\psi_{j, \epsilon}^{0}\left(\right.$ respectively $\left.\psi_{j, \epsilon}^{\infty}\right)$ and Lavaurs linear maps.

Typically, a renormalized return in $w$-coordinate is of one of the forms

$$
\left\{\begin{array}{l}
L_{c_{s}(\epsilon)} \circ \psi_{j_{s}, \epsilon}^{0} \circ \cdots \circ L_{c_{1}(\epsilon)} \circ \psi_{j_{1}, \epsilon}^{0}, \\
L_{c_{s}(\epsilon)} \circ \psi_{j_{s}, \epsilon}^{\infty} \circ \cdots \circ L_{c_{1}(\epsilon)} \circ \psi_{j_{1}, \epsilon}^{\infty}, \\
\psi_{j_{s}, \epsilon}^{0} \circ L_{c_{s}(\epsilon)} \circ \cdots \circ \psi_{j_{1}, \epsilon}^{0} \circ L_{c_{1}(\epsilon)}, \\
\psi_{j_{s}, \epsilon}^{\infty} \circ L_{c_{s}(\epsilon)} \circ \cdots \circ \psi_{j_{1}, \epsilon}^{\infty} \circ L_{c_{1}(\epsilon)} .
\end{array}\right.
$$

If $\lambda=\exp (\mu)$ is the multiplier of $f_{\epsilon}$ at one fixed point, then the multiplier at the fixed point of the renormalized return map in $w$ coordinate is simply $\lambda^{\prime}=\exp \left(4 \pi^{2} / \mu\right)$.

The parametric resurgence phenomenon occurs when $\lambda^{\prime}$ is resonant: $\lambda^{\prime}=\exp (2 \pi i p / q)$. Note that this can occur along sequences $\left\{\epsilon_{n}\right\}$ of parameter values converging to 0 , corresponding, for instance, to $\mu_{n}=-2 \pi i q /(p+n q)$, which are located inside an open sector $W_{\ell}$, and for which the constant $c\left(\epsilon_{n}\right)$ in the Lavaurs linear maps have a limit $c(0)$ when $n \rightarrow \infty$. Then, on such a sequence $\left\{\epsilon_{n}\right\}$ of parameter values, the coefficients of the normal form converge to the coefficients of the normal form of

$$
\left\{\begin{array}{l}
L_{c_{s}(0)} \circ \psi_{j_{s}, 0}^{0} \circ \cdots \circ L_{c_{1}(0)} \circ \psi_{j_{1}, 0}^{0}, \\
L_{c_{s}(0)} \circ \psi_{j_{s}, 0}^{\infty} \circ \cdots \circ L_{c_{1}(0)} \circ \psi_{j_{1}, 0}^{\infty}, \\
\psi_{j_{s}, 0}^{0} \circ L_{c_{s}(0)} \circ \cdots \circ \psi_{j_{1}, 0}^{0} \circ L_{c_{1}(0)}, \\
\psi_{j_{s}, 0}^{\infty} \circ L_{c_{s}(0)} \circ \cdots \circ \psi_{j_{1}, 0}^{\infty} \circ L_{c_{1}(0)} .
\end{array}\right.
$$

This implies that if one of these maps is nonlinearizable of order $m$ (because of the nonvanishing of the $m$ th nonlinear term in the normal form) then, for $n$ sufficiently large, the corresponding renormalized map is nonlinearizable of order $m^{\prime}\left(\epsilon_{n}\right) \leq m$. As a general rule, the parametric resurgence phenomenon concerns cases where the only way that there can be some incompatibility between the different sectorial normal forms on the different $V_{j, \epsilon}^{ \pm}$ is for the fixed points themselves to be nonlinearizable.

Applications in conformal geometry in the case $k=1$ appear in [12] and [14].

Acknowledgements. The author is very grateful to Colin Christopher for stimulating discussions and helpful suggestions. The author thanks the referee for careful reading and fine remarks. This work is supported by NSERC in Canada.

\section{REFERENCES}

[1] C. Christopher and C. Rousseau. The moduli space of germs of generic families of analytic diffeomorphisms unfolding a parabolic fixed point. Preprint, 2008, arXiv:0809.2167. Int. Math. Res. Not. to appear.

[2] A. Douady and P. Sentenac. Champs de vecteurs polynomiaux sur $\mathbb{C}$. Preprint, 2005. 
[3] A. A. Glutsyuk. Confluence of singular points and nonlinear Stokes phenomenon. Trans. Moscow Math. Soc. 62 (2001), 49-95.

[4] Y. Ilyashenko (Ed.). Nonlinear Stokes phenomena. Nonlinear Stokes Phenomena (Advances in Soviet Mathematics, 14). American Mathematical Society, Providence, RI, 1993, pp. 1-55.

[5] V. Kostov. Versal deformations of differential forms of degree $\alpha$ on the line. Funct. Anal. Appl. 18 (1984), 335-337.

[6] C. Lambert and C. Rousseau. Complete system of analytic invariants for unfolded differential linear systems with an irregular singularity of Poincaré rank one. Mosc. Math. J. 12 (2012), 77-138.

[7] P. Mardešić, R. Roussarie and C. Rousseau. Modulus of analytic classification for unfoldings of generic parabolic diffeomorphisms. Mosc. Math. J. 4 (2004), 455-498.

[8] J. Martinet. Remarques sur la bifurcation nœud-col dans le domaine complexe. Astérisque 150-151 (1987), 131-149.

[9] R. Oudkerk. The parabolic implosion for $f_{0}(z)=z+z^{v+1}+O\left(z^{v+2}\right)$. PhD Thesis, University of Warwick, 1999.

[10] C. Rousseau. Addendum to modulus of analytic classification for unfoldings of generic parabolic diffeomorphisms. Mosc. Math. J. 4 (2004), 499-502.

[11] C. Rousseau. Modulus of orbital analytic classification for a family unfolding a saddle-node. Mosc. Math. J. 5 (2005), 245-268.

[12] C. Rousseau. The root extraction problem. J. Differential Equations 234 (2007), 110-141.

[13] C. Rousseau. The moduli space of germs of generic families of analytic diffeomorphisms unfolding of a codimension one resonant diffeomorphism or resonant saddle. J. Differential Equations 248 (2010), 1794-1825.

[14] C. Rousseau. The modulus of unfoldings of cusps in conformal geometry. J. Differential Equations 252 (2012), 1562-1588.

[15] C. Rousseau and C. Christopher. Modulus of analytic classification for the generic unfolding of a codimension one resonant diffeomorphism or resonant saddle. Ann. Inst. Fourier (Grenoble) 57 (2007), 301-360.

[16] C. Rousseau and L. Teyssier. Analytical moduli for unfoldings of saddle-node vector-fields. Mosc. Math. J. 8 (2008), 547-616.

[17] M. Shishikura. Bifurcations of parabolic fixed points. The Mandelbrot Set, Theme and Variations (London Mathematical Society Lecture Note Series, 274). Ed. Tan Lei. Cambridge University Press, Cambridge, 2000, pp. 325-363. 